\title{
Clinical application of chromosomal microarray for pathogenic genomic imbalance in fetuses with increased nuchal translucency but normal karyotype
}

\author{
Dongsook Lee ${ }^{\oplus}$, Sanghee $\mathrm{Go}^{\oplus}$, Sohyun $\mathrm{Na}^{\oplus}$, Surim Park ${ }^{\oplus}$, Jinyoung Ma ${ }^{\oplus}$, and Doyeong Hwang*๑ \\ Research Center of Fertility \& Genetics, Hamchoon Women's Clinic, Seoul, Korea
}

\begin{abstract}
Purpose: To evaluate the additive value of prenatal chromosomal microarray analysis (CMA) in assessing increased nuchal translucency (NT) $(\geq 3.5 \mathrm{~mm}$ ) with normal karyotype and the possibility of detecting clinically significant genomic imbalance, based on specific indications.

Materials and Methods: Invasive samples from 494 pregnancies with NT $\geq 3.5 \mathrm{~mm}$, obtained from the Research Center of Fertility \& Genetics of Hamchoon Women's Clinic between January 2019 and February 2020, were included in this study and CMA was performed in addition to a standard karyotype.

Results: In total, 494 cases were subjected to both karyotype and CMA analyses. Among these, 199 cases of aneuploidy were excluded. CMA was performed on the remaining 295 cases (59.7\%), which showed normal (231/295, 78.3\%) or non-significant copy number variation (CNV), such as benign CNV or variants of uncertain clinical significance likely benign (53/295, 18.0\%). Clinically significant CNVs were detected in 11 cases (11/295, 3.7\%).

Conclusion: Prenatal CMA resulted in a 3\% to $4 \%$ higher CNV diagnosis rate in fetuses exhibiting increased NT ( $\geq 3.5 \mathrm{~mm}$ ) without other ultrasound detected anomalies and normal karyotype. Therefore, we suggest using high resolution, non- targeting CMA to provide valuable additional information for prenatal diagnosis. Further, we recommend that a genetics specialist should be consulted to interpret the information appropriately and provide counseling and follow-up services after prenatal CMA.
\end{abstract}

Key words: Nuchal translucency measurement, Increased nuchal translucency $(\geq 3.5 \mathrm{~mm})$, Prenatal chromosomal microarray, Prenatal chromosomal microarray analysis.

\section{Introduction}

Nuchal translucency (NT) is the thickness of fluid collection in the fetal neck. This may be observed by an ultrasound scan, that is performed between 10 and 13 weeks 6 days of gestation. An increased NT ( $\geq 3.5 \mathrm{~mm}$, or $>99$ th percentile) is correlated not only with chromosomal aneuploidies, but also with important defects of the heart and arteries, skeletal dysplasia to a considerable extent, as well as some genetic syndromes. In addition, when these defects occur, there is an increased risk of miscarriage, intrauterine fetal death, or delayed development $[1,2]$.

Cytogenetic analysis of amniotic fluid (AF) or chorionic villi

Received: 24 May 2020, Revised: 14 June 2020, Accepted: 15 June 2020, Published: 30 June 2020

${ }^{*}$ Corresponding author: Doyeong Hwang, M.D., Ph.D. iD https://orcid.org/https://orcid.org/0000-0002-1452-9023

Research Center of Fertility \& Genetics, Hamchoon Women's Clinic, 10 Seochojungang-ro 8-gil, Seocho-gu, Seoul 06643, Korea.

Tel: +82-2-522-0123, Fax: +82-2-522-2388, E-mail: doyhwang@hamchoon.com

Conflict of interest: The authors declare that they do not have any conflicts of interest.

(C) This is an open-access article distributed under the terms of the Creative Commons Attribution Non-Commercial License (http://creativecommons.org/licenses/by-nc/4.0/) which permits unrestricted non-commercial use, distribution, and reproduction in any medium, provided the original work is properly cited.

(c) Copyright 2020 by the Korean Society of Medical Genetics and Genomics 
samples (CVS) has been the gold standard prenatal diagnostic method for the detection of abnormal karyotypes. This method reliably analyzes chromosomal aneuploidies, as well as structural abnormalities. Chromosomal microarray analysis (CMA) is a novel method that detects not only the chromosomal aneuploidies, but also the copy number variations (CNVs). These CNVs appear in genomic imbalances, such as microdeletions and microduplications, which may be correlated with known genetic syndromes, or abnormal clinical phenotypes [3,4].

The latest meta-analysis, regarding the need for CMA in cases with increased NT and normal karyotype, showed progressive diagnosis rates between 4\% (increased NT) and 7\% (complex ultrasound malformation), including aberrations that involved $22 q 11.2[5,6]$.

Most researchers have proposed CMA for prenatal diagnosis, in cases with NT $\geq 3.5 \mathrm{~mm}$. Also, the increased resolution achieved by CMA provides a broader scope to diagnose chromosomal aberrations, as well as an increase in the number of uncertain findings, secondary findings, or adult-onset disease indicators. Uncertain findings or secondary results are the major dilemmas in prenatal genetic counseling. In our study, we aimed to evaluate the clinical interpretations of prenatal $\mathrm{CMA}_{\text {, for the }}$ inspection of genomic imbalances in specimens from fetuses with an increased NT ( $\geq 3.5 \mathrm{~mm}$ ) and a normal karyotype [7-9].

\section{Materials and Methods}

The invasive samples from 494 pregnant women with fetuses having a NT $\geq 3.5 \mathrm{~mm}$ included in this study were obtained from the Research Center of Fertility \& Genetics of Hamchoon Women's Clinic between January 2019 and February 2020. These were subjected to prenatal diagnosis using rapid aneuploidy detection (RAD), G-band karyotyping and CMA. Details regarding the analysis flow are shown in Fig. 1.

All the samples underwent either quantitative fluorescence polymerase chain reaction (QF-PCR) or the direct method for RAD. Conventional cytogenetic analysis was carried out on CVS. This was in accordance with the standard protocols used for examining the numerical and structural aberrations of chromosomes, in direct cytotrophoblastic cell-preparations and longterm cultures of mesenchymal tissue (GTG-banding, 550 band level). Cytogenetic analysis using AF was also carried out according to the standard protocols.

Genomic DNA for prenatal CMA was extracted from T25flask-cultured fetal cells using a Qiagen DNA mini kit (Qiagen, Valencia, CA, USA), according to the manufacturer's protocol. For single nucleotide polymorphism (SNP) array analysis, the genomic DNA was screened using CytoScan 750K (Affymetrix Inc., Santa Clara, CA, USA), and analyzed using Affymetrix gene chip kit software ChAS 3.2. Our CNV results were compared with those in public CNV databases (Database of Genomic Variants [http://projects.tcag.ca/variation/]; Decipher [http://decipher.

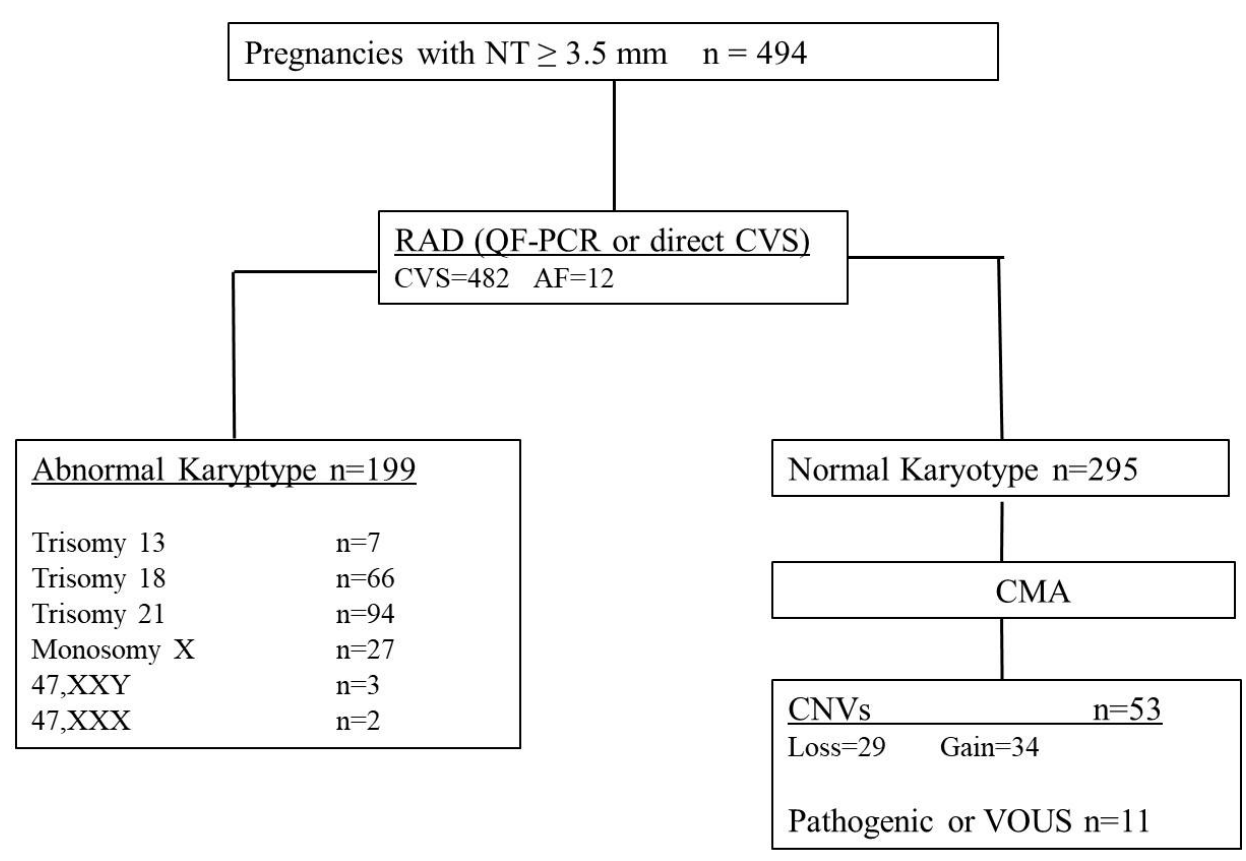

Fig. 1. Characteristics of pregnant women and aberrant CMA findings in fetuses with increased NTs $(\geq 3.5 \mathrm{~mm})$. CMA, chromosomal microarray; NT, nuchal translucency; RAD, rapid aneuploidy detection; QF$\mathrm{PCR}$, quantitative fluorescence polymerase chain reaction; CNVs, copy number variantions; AF, amniotic fluid; VOUS, variants of uncertain clinical significance. 


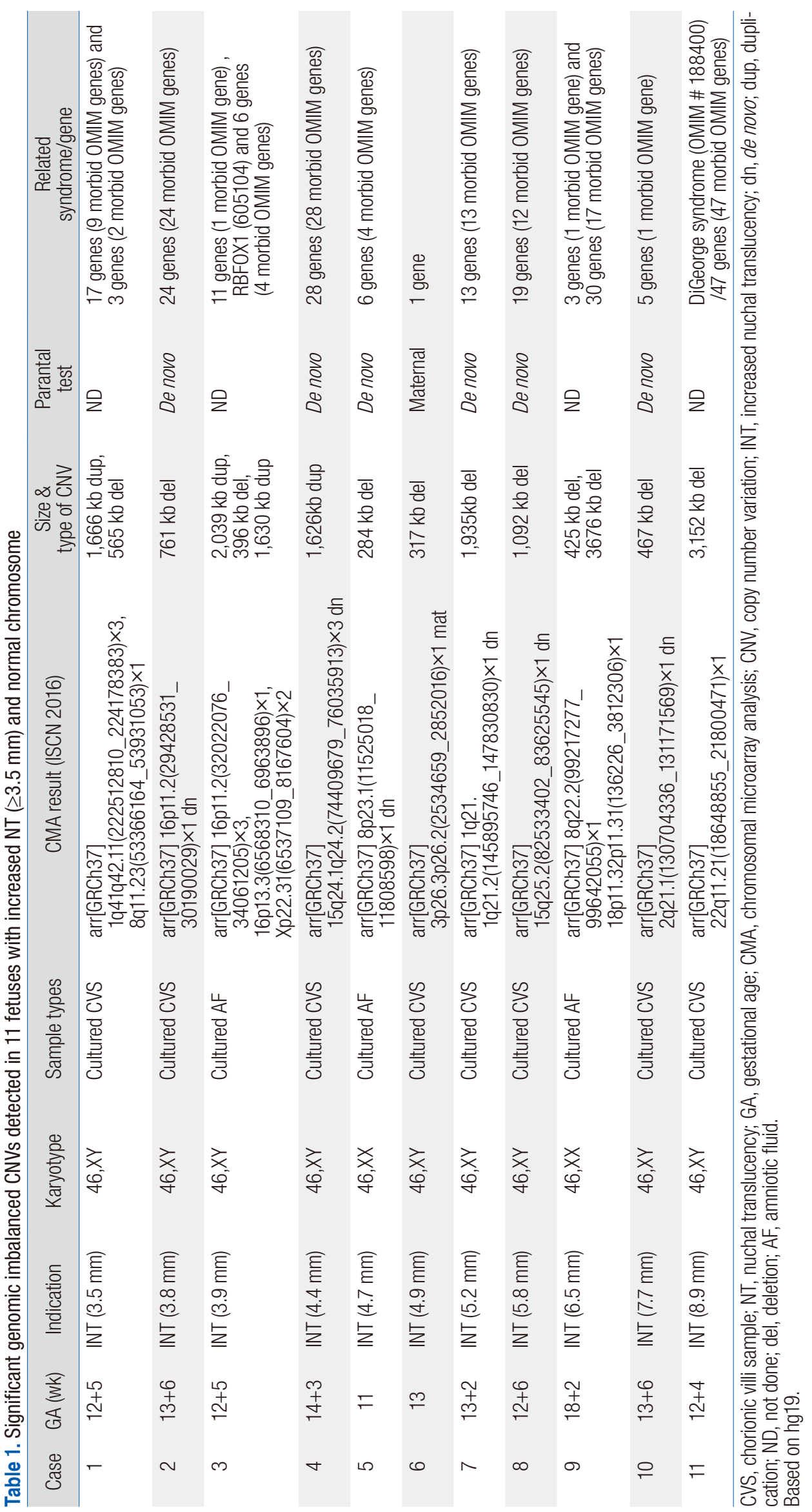


sanger.ac.uk]; ISCA[https://www.iscaconsortium.org]; UCSC [http://genome.ucsc.edu/]; OMIM [http://www.omim.org/]), by trained investigators. Further, they were classified as pathogenic, likely pathogenic, uncertain clinical significance, likely benign, or benign according to the guidelines of the American College of Medical Genetics [10]. CNVs were reported to the physician according to the guidelines of the Society of Obstetricians and Gynaecologists of Canada (SOGC)-Canadian College of Medical Geneticists (CCMG) [5]. If a pathogenic sample was detected, parental CMA testing was recommended in order to confirm the aberration. Also, eventually, it was reconfirmed via secondary genetic methods such as fluorescence in situ hybridization or multiplex ligation-dependent probe amplification (MLPA).

\section{Results}

Fig. 1 summarizes the fetuses examined in this study. Out of the 494 pregnancies with increased fetal NT at 10 to 13 weeks 6 days of gestation that were analyzed in the study, the invasive procedures performed were CVS (482/494, 97.6\%) or amniocentesis (12/494, 2.4\%). Rapid aneuploidy testing (QF-PCR, direct CVS) showed 199 (40.3\%) cases having an aneuploidy involving chromosomes $13,18,21$ or $X$, consistent with the karyotype analyzed in the cultured fetal tissue. In total, 494 cases were subjected to both karyotype and CMA analyses, among which 199 cases of aneuploidy were excluded. The remaining 295 (59.7\%) cases underwent CMA, and had a normal CMA result (231/295, 78.3\%), or showed a non-significant CNV, such as benign CNV or VOUS likely benign (53/295, 18.0\%). Clinically significant CNVs were detected in 11 cases (11/295, 3.7\%) (Table 1). The size of the $C N V$, its genomic position, and gene content were re-evaluated for clinical significance by referencing the latest published studies and public databases. Table 1 shows the list of cases with clinically significant genomic imbalances. Out of seven cases (Cases 2, 4-8, 10), six were de novo and one was inherited from the mother; in four cases (Cases 1, 3, 9, 11), the parents were not tested; however, out of three of these cases (Cases 1, 3, 11), one had an abnormal ultrasound finding in addition to the NT. Case 11 (DiGeorge syndrome) was reconfirmed with MLPA P372-B1 (Fig. 2) and matched.

\section{Discussion}

In our study, 3.7\% of the fetuses had a significantly increased NT ( $\geq 3.5 \mathrm{~mm}$; 99th percentile); however, through CMA, even the normal karyotypes were found to have pathogenic genomic imbalances. This incidence was very similar to the guidelines of the 2011 SOGC-CCMG [5]. Therefore, our results corroborate that prenatal CMA identifies clinically significant CNVs, that are not detectable by conventional cytogenetic methods. The most frequent pathogenic CNVs that were related to increased NT were 22q11.2 microdeletions/microduplications. We previously reported the detection of 22q11.2 genomic imbalance by the MLPA method, which was used even though the karyotype was normal, despite the increased NT [11].

To date, karyotype analysis remains a reliable method in prenatal diagnosis. However, because of the limitations of chromosomal analysis, most researchers discuss the benefits

(A)

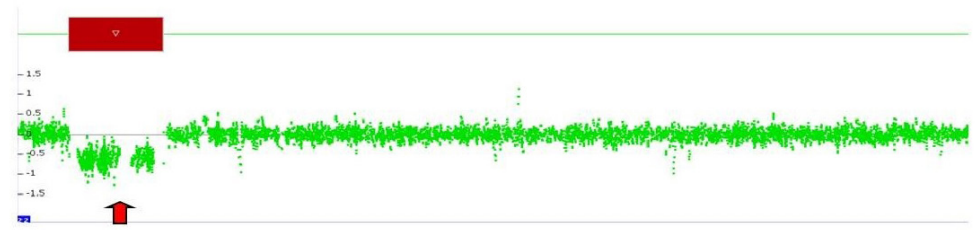

22q11.21(18648855_21800471)x1

(B)

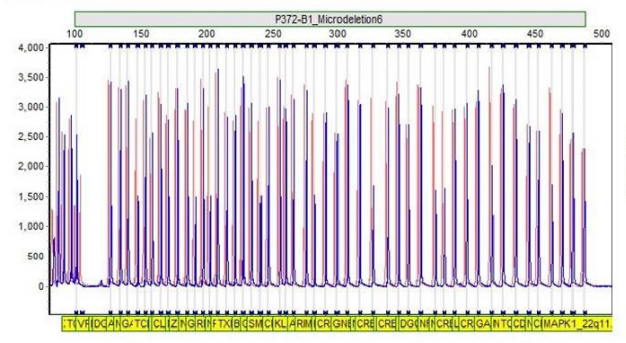

Fig. 2. Case 11 results in deleted 22q11.21. (A) CMA image showing a 3.152 kbp deletion. (B) MLPA results in the MRCHolland P372-B1. 
and limitations of using CMA and conventional cytogenetic methods for prenatal diagnostic testing of pregnant women, and propose additional options for CMA. CMA method has a higher resolution than conventional karyotyping, and is capable of diagnosing smaller submicroscopic imbalances, including the uniparental disomy and loss of heterozygosity, by SNP array $[8,9,12]$. For fetuses having an increased risk of submicroscopic chromosomal imbalances, such as those with increased NT, the utility of the supplemental data provided by CMA in prenatal diagnosis is evident, and helps in reducing the rate of undiagnosed diseases, or the likelihood of their occurrence. CMA could provide more accurate predictive perceptions as compared to karyotype analysis, thereby affecting pregnancy management and the concomitant outcomes.

Nevertheless the interpretation of CMAs and the policy for classifying CNVs is challenging. In our study, we noticed certain pathogenic variants or likely pathogenic variants, as they do not always correspond to severe defects, and may be inherited from a parent having very little or no clinical features. Therefore, it is suggested that the CMA must be interpreted with caution in prenatal diagnosis, by using proper guidelines. Our team interpreted the results based on the guidelines of the SOGC-CCMG, and informed the pregnant women and their families.

For clinically significant imbalances (pathogenic), it is necessary to carry out parental testing. Due to incomplete penetrance and a variable phenotype, these CNVs also raised concerns and frustration among parents, which lead to confusion regarding the future health and development of the offspring. In the present study, among seven cases (Cases 2, 4-8, 10) [13-19], six were de novo, while one was maternally inherited; in four cases (Cases 1, 3, 9, 11) [20-23], the parents were not tested; however, from three of the (Cases 1, 3, 11) cases, one was observed with an abnormality in the ultrasound, in addition to the NT. Nevertheless, the clinical significance of these variants cannot be predicted before parental confirmation. CMA was inadequate for accurately indicating the clinical significance of a formerly unreported CNV. Instead, parental confirmation is necessary in order to rule out some CNVs $[24,25]$, which tend to be benign. Fortunately, with the application of CMA and sustained improvements in the data from the database, the incidence of VOUSs can be reduced. It is advisable to reconfirm the findings using other genetic diagnostic methods, if possible.

We encountered both counseling challenges and ethical dilemmas with the initiation of prenatal CMA. These challenges included cases that were classified into: VOUS, CNVs with incomplete penetrance, and conditions with the onset of adult- hood. Clearly, such cases require additional extensive counseling, as opposed to informing a patient about a test result being normal or abnormal, which is the case with karyotype results. A key aspect in such cases is comprehensive genetic counseling by an expert, who is a specialist in interpreting prenatal CMA information.

In our study, CMA showed a 3\% to 4\% increase in the number of CNV diagnosed fetuses, with increased NT ( $\geq 3.5 \mathrm{~mm}$ ), and an absence of other ultrasound anomalies and normal karyotype. Therefore, we suggest using a high resolution, non-targeting CMA to provide useful additional information for prenatal diagnosis. A key aspect in such cases is comprehensive genetic counseling by an experienced provider, having a specialization in interpreting the prenatal CMA. Also, we recommend involving of a genetics specialist service in prenatal CMA interpretation, counseling, and follow-up.

\section{References}

1. Egloff $M$, Hervé $B$, Quibel $T$, Jaillard $S$, Le Bouar $G$, Uguen $K$, et al. Diagnostic yield of chromosomal microarray analysis in fetuses with isolated increased nuchal translucency: a French multicenter study. Ultrasound Obstet Gynecol 2018;52:715-21.

2. Maya I, Yacobson S, Kahana S, Yeshaya J, Tenne T, Agmon-Fishman I, et al. Cut-off value of nuchal translucency as indication for chromosomal microarray analysis. Ultrasound Obstet Gynecol 2017;50:3325.

3. Bornstein E, Berger $S$, Cheung SW, Maliszewski KT, Patel A, Pursley $A N$, et al. Universal prenatal chromosomal microarray analysis: additive value and clinical dilemmas in fetuses with a normal karyotype. Am J Perinatol 2017;34:340-8.

4. Cicatiello R, Pignataro P, Izzo A, Mollo N, Pezone L, Maruotti GM, et al. Chromosomal microarray analysis versus karyotyping in fetuses with increased nuchal translucency. Med Sci (Basel) 2019;7:40.

5. Armour CM, Dougan SD, Brock JA, Chari R, Chodirker BN, DeBie I, et al.; On-Behalf-Of the Canadian College of Medical Geneticists. Practice guideline: joint CCMG-SOGC recommendations for the use of chromosomal microarray analysis for prenatal diagnosis and assessment of fetal loss in Canada. J Med Genet 2018;55:215-21.

6. Breman A, Pursley AN, Hixson P, Bi W, Ward P, Bacino CA, et al. Prenatal chromosomal microarray analysis in a diagnostic laboratory; experience with $>1000$ cases and review of the literature. Prenat Diagn 2012;32:351-61.

7. Stosic M, Levy B, Wapner R. The use of chromosomal microarray analysis in prenatal diagnosis. Obstet Gynecol Clin North Am 2018:45:55-68. 
8. Su L, Huang H, An G, Cai M, Wu X, Li Y, et al. Clinical application of chromosomal microarray analysis in fetuses with increased nuchal translucency and normal karyotype. Mol Genet Genomic Med 2019;7:e811.

9. Zhao XR, Gao L, Wu Y, Wang YL. Application of chromosomal microarray in fetuses with increased nuchal translucency. J Matern Fetal Neonatal Med 2020;33:1749-54.

10. South ST, Lee $C_{1}$ Lamb AN, Higgins AW, Kearney HM; Working Group for the American College of Medical Genetics and Genomics Laboratory Quality Assurance Committee. ACMG Standards and Guidelines for constitutional cytogenomic microarray analysis, including postnatal and prenatal applications: revision 2013. Genet Med 2013;15:9019.

11. Lee D, Na S, Park S, Go S, Ma J, Yang S, et al. Clinical experience with multiplex ligation-dependent probe amplification for microdeletion syndromes in prenatal diagnosis: 7522 pregnant Korean women. Mol Cytogenet 2019;12:10.

12. Leung TY, Au Yeung KC, Leung WC, Leung KY, Lo TK, To WWK, et al. Prenatal diagnosis of pathogenic genomic imbalance in fetuses with increased nuchal translucency but normal karyotyping using chromosomal microarray. Hong Kong Med J 2019;25 Suppl 5:30-2.

13. Shinawi M, Liu P, Kang SH, Shen J, Belmont JW, Scott DA, et al. Recurrent reciprocal 16p11.2 rearrangements associated with global developmental delay, behavioural problems, dysmorphism, epilepsy, and abnormal head size. J Med Genet 2010;47:332-41.

14. Chen CP, Chen CY, Chern SR, Wu PS, Chen SW, Lai ST, et al. Molecular cytogenetic characterization of a duplication of 15q24.2-q26.2 associated with anencephaly and neural tube defect. Taiwan J Obstet Gynecol 2017;56:550-3.

15. Wat MJ, Shchelochkov OA, Holder AM, Breman AM, Dagli A, Bacino C, et al. Chromosome 8p23.1 deletions as a cause of complex congenital heart defects and diaphragmatic hernia. Am J Med Genet A 2009;149A:1661-77.

16. Fernandez T, Morgan T, Davis N, Klin A, Morris A, Farhi A, et al. Disruption of contactin 4 (CNTN4) results in developmental delay and other features of 3p deletion syndrome. Am J Hum Genet 2004;74:128693.

17. Gamba BF, Zechi-Ceide RM, Kokitsu-Nakata NM, Vendramini-Pittoli S, Rosenberg C, Krepischi Santos AC, et al. Interstitial 1q21.1 microdeletion is associated with severe skeletal anomalies, dysmorphic face and moderate intellectual disability. Mol Syndromol 2016;7:344-8.

18. Burgess T, Brown NJ, Stark Z, Bruno DL, Oertel R, Chong B, et al. Characterization of core clinical phenotypes associated with recurrent proximal 15q25.2 microdeletions. Am J Med Genet A 2014;164A:7786.

19. Dharmadhikari AV, Kang SH, Szafranski P, Person RE, Sampath $S$, Prakash SK, et al. Small rare recurrent deletions and reciprocal duplications in 2q21.1, including brain-specific ARHGEF4 and GPR148. Hum Mol Genet 2012;21:3345-55.

20. Weise A, Mrasek K, Klein E, Mulatinho M, Llerena JC Jr, Hardekopf D, et al. Microdeletion and microduplication syndromes. J Histochem Cytochem 2012;60:346-58.

21. Lal D, Trucks $H$, Møller RS, Hjalgrim H, Koeleman BP, de Kovel CG, et al.; EMINet Consortium; EPICURE Consortium. Rare exonic deletions of the RBFOX1 gene increase risk of idiopathic generalized epilepsy. Epilepsia 2013;54:265-71.

22. Miller DT, Adam MP, Aradhya S, Biesecker LG, Brothman AR, Carter $N P$, et al. Consensus statement: chromosomal microarray is a firsttier clinical diagnostic test for individuals with developmental disabilities or congenital anomalies. Am J Hum Genet 2010;86:749-64.

23. Burnside RD. 22q11.21 Deletion syndromes: a review of proximal, central, and distal deletions and their associated features. Cytogenet Genome Res 2015;146:89-99.

24. Huang LY, Pan M, Han J, Zhen L, Yang $X$, Li DZ. What would be missed in the first trimester if nuchal translucency measurement is replaced by cell free DNA foetal aneuploidy screening? J Obstet Gynaecol 2018;38:498-501.

25. Lund IC, Christensen R, Petersen OB, Vogel I, Vestergaard EM. Chromosomal microarray in fetuses with increased nuchal translucency. Ultrasound Obstet Gynecol 2015;45:95-100. 